\title{
Spin-polarized electron tunneling across magnetic dielectric
}

\author{
I. V. Shvets ${ }^{a}$ \\ SFI Nanoscience Laboratories, Physics Department, Trinity College, Dublin 2, Ireland
}

\author{
A. N. Grigorenko and K. S. Novoselov \\ Department of Physics and Astronomy, University of Manchester, Manchester, M13 9PL, United Kingdom \\ D. J. Mapps \\ Department of Communication, Electronic and Electrical Engineering, University of Plymouth, Plymouth, \\ PL4 8AA, United Kingdom
}

(Received 9 February 2005; accepted 25 March 2005; published online 16 May 2005)

\begin{abstract}
This letter deals with a magnetic tunnel junction having spin filtering by a magnetic barrier. We performed experiments in which a relatively strong external field rotates magnetizations of both ferromagnetic electrodes in the tunnel junction with the magnetic barrier simultaneously so that the two are always parallel to each other. The tunnel magnetoresistance induced in this way was over $16 \%$ at $300 \mathrm{~K}$. The angular dependency of the tunnel current on the layer magnetizations indicates that the barrier contains antiferromagnetic oxide. To achieve the described effect the magnetic electrode of the junction was oxidized prior to forming the $\mathrm{Al}_{2} \mathrm{O}_{3}$ layer. (C) 2005 American Institute of Physics. [DOI: 10.1063/1.1925785]
\end{abstract}

Conventional spin-polarized electron tunneling is based on a tunnel junction with two ferromagnetic electrodes. ${ }^{1-5}$ The tunnel current between the electrodes depends on their relative orientations of magnetization with respect to each other. ${ }^{6}$ Spin-dependent currents can also be achieved in the case of tunneling between an antiferromagnetic electrode and a ferromagnetic one. In this case, the tunnel current changes when the magnetization vector in the ferromagnetic electrode rotates with respect to the antiferromagnetic direction of the other electrode. $^{7-9}$

Another approach to achieving spin-dependent tunneling was proposed in Ref. 10. To explain the concept, let us consider two ferromagnetic electrodes separated by a tunnel barrier. We assume that magnetizations $\mathbf{M}_{1}$ and $\mathbf{M}_{2}$ in both electrodes are always parallel. Contrary to the conventional approach, however, we consider the tunnel barrier composed of a ferromagnetic dielectric. The direction of magnetization $\mathbf{M}_{b}$ in the barrier is different from the direction of magnetizations $\mathbf{M}_{1}$ and $\mathbf{M}_{2}$ in the electrodes. In this case, the tunnel current between the two ferromagnetic electrodes, depends on the relative direction of the spin of electrons emitted by the electrodes, with respect to the magnetization of the tunnel barrier, because the tunneling electrons see interaction with the spins of the dielectric layer through an additional exchange energy $-J \boldsymbol{\sigma}_{1} \boldsymbol{\sigma}_{b}$, where $J$ is the exchange constant and $\hbar \boldsymbol{\sigma}_{1} / 2$ and $\hbar \boldsymbol{\sigma}_{b} / 2$ are electron spins in the first electrode and barrier, respectively. This either increases or decreases the effective tunnel barrier depending on the relative direction of spins in the electrodes and the ferromagnetic layer. Therefore, the tunnel current at low bias voltage $V$ is

$$
I=V G \exp \left(-\frac{2 d \sqrt{2 m(\phi \mp J)}}{\hbar}\right),
$$

where signs - and + in Eq. (1) correspond to the cases of the spin directions in the tunnel barrier and in the electrodes

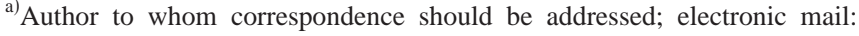
ivchvets@tcd.ie
}

being parallel and antiparallel to each other, respectively, provided $J$ is positive (ferromagnetic exchange) and vice versa for negative $J . G$ is the barrier conductivity per unit area, $m$ is the free electron mass, $\phi$ is the barrier height, $d$ is the barrier width. In writing Eq. (1), we assume 100\% spin polarization at the Fermi level. If $J \ll \phi$, then the relative change of the conductivity is

$$
\Delta G /\langle G\rangle=2 \tanh \left(k_{\mathrm{eff}} d\right),
$$

where $k_{\text {eff }}=J \sqrt{2 m} /(\hbar \sqrt{\phi})$ and $\langle G\rangle$ is the average conductivity. If we substitute in these formulae some typical values of the barrier width $d=1 \mathrm{~nm}$, the barrier height $\phi=4 \mathrm{eV}$ and the exchange $J=0.1 \mathrm{eV}$, we obtain $k_{\text {eff }}=0.29 \mathrm{~nm}^{-1}$ and tunneling magnetoresistance (TMR) of $55 \%(\mathrm{TMR}=\Delta R / R$, where $R$ $=1 / G$ is the junction resistance). When the effective electrode spin polarizations at Fermi level is below unity, $P_{1(2)}$ $<1$, and for arbitrary angles between quantization axes of the first electrode and the barrier, $\chi$, and the second electrode and barrier, $\varphi$, the same considerations yield the tunnel conductivity as

$$
\begin{aligned}
G= & G_{0}\left[\left[1+P_{1} P_{2} \cos (\theta)\right] \cosh \left(k_{\mathrm{eff}} d\right)\right. \\
& \left.+\left[P_{1} \cos (\chi)+P_{2} \cos (\varphi)\right] \sinh \left(k_{\mathrm{eff}} d\right)\right],
\end{aligned}
$$

where $\theta$ is the angle between quantization axes of electrodes. The second term describes the discussed effect (with $\chi=-\varphi$ ) and disappears for $J=0\left(k_{\text {eff }}=0\right)$.

A similar scheme can be used when the tunnel barrier is an antiferromagnetic dielectric layer sandwiched between two ferromagnetic electrodes (Fig. 1). In this case, the spin operator of barrier electrons $\mathbf{S}_{b}$ is replaced by the antiferromagnetic operator $\mathbf{L}_{b}$ and the angular dependency of the current on the direction of magnetization in ferromagnetic electrodes should reflect the symmetry of the antiferromagnetic spin order.

This approach utilizing the magnetic barrier is rather uncommon with only very small number of studies dedicated to it. ${ }^{11}$ The difficulty with implementing the method proposed is that there is not a wide choice of materials that are known to 

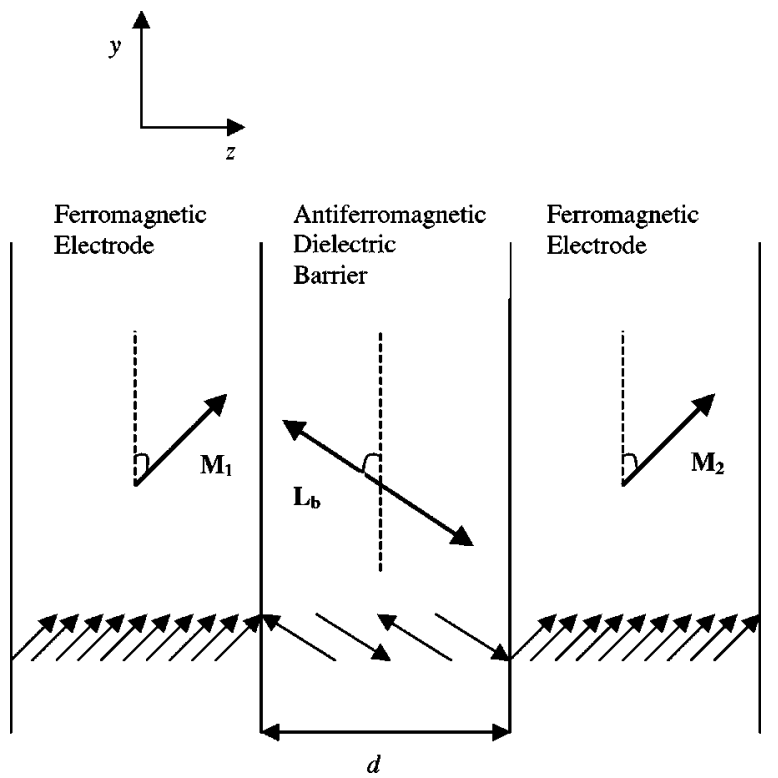

FIG. 1. Schematics of the tunnel junction with two ferromagnetic electrodes and antiferromagnetic tunnel barrier.

be suitable for fabricating uniform tunnel barrier layers. Essentially almost the entire body of work with tunnel junctions so far was done with barriers of $\mathrm{Al}_{2} \mathrm{O}_{3}$ and $\mathrm{MgO}$. Therefore, we employed the approach of oxidizing the electrodes of transition metals and then forming the $\mathrm{Al}_{2} \mathrm{O}_{3}$ layer on top of the layer of the transition metal oxide. The expectation is that even though the transition metal oxide may not be uniform, the magnetic oxide will cover a significant area of the tunnel junction and the leakage of current through the pinholes will be suppressed by the layer of conventional tunnel barrier material.

The $\mathrm{NiFe} / \mathrm{Al}_{2} \mathrm{O}_{3} / \mathrm{Co}$ and $\mathrm{NiFe} / \mathrm{Al}_{2} \mathrm{O}_{3} / \mathrm{Co}(\mathrm{CoFe})$ junctions have been fabricated on a silicon substrate in a Nordico2000 system and patterned using optical lithography. We have induced anisotropies in the magnetic layers by permanent magnets placed in the substrate holder during deposition. This procedure and the characteristics of the anisotropy induced are described in detail in Ref. 12. The direction of anisotropy in both ferromagnetic layers was the same. The interlayer coupling and demagnetization factors were small for the studied junctions. Figure 2(a) shows the magnetization curves for the representative circular junction (radius $500 \mu \mathrm{m}$ ) with the parallel anisotropies, measured for the magnetic field applied at an angle of $3^{\circ}$ with respect to the anisotropy axis. This figure demonstrates that the typical magnetization switching field is in the range of some 2-15 Oe. We did not measure the magnetization curves and the in-plane anisotropies in the smaller junctions directly. These anisotropies and coupling between layers have been extracted from the TMR response. The corresponding anisotropy field values were 4-7 Oe in NiFe layer and 14-20 Oe in $\mathrm{Co}, \mathrm{Co}(\mathrm{CoFe})$ layers and were consistent with the magnetization values taken on larger size junctions. The intrinsic junction resistance scaled inversely with the junction area and was about $200 \Omega \times \mu \mathrm{m}^{2}$ at low bias for the $\mathrm{NiFe} / \mathrm{Al}_{2} \mathrm{O}_{3} / \mathrm{Co} \quad$ structure $\quad\left[17 \Omega \times \mu \mathrm{m}^{2}\right.$ for the $\mathrm{NiFe} / \mathrm{Al}_{2} \mathrm{O}_{3} / \mathrm{Co}(\mathrm{CoFe})$ structure] with the maximal roomtemperature TMR produced by the switching of the soft magnetic layer of about $\mathrm{TMR}_{\max }=15 \%$. All of the junctions showed similar behavior in respect to the angular depen-
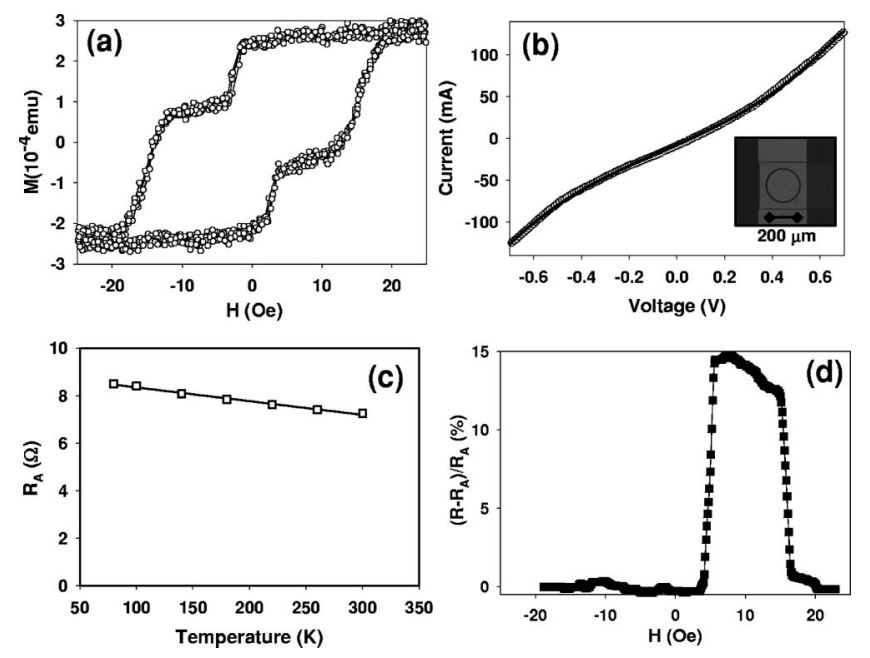

FIG. 2. (a) Magnetization curves for the circular $\mathrm{NiFe} / \mathrm{Al}_{2} \mathrm{O}_{3} / \mathrm{Co}$ junction of $500 \mu \mathrm{m}$ in radius. (b) The $I-V$ characteristic of the junction with the radius $100 \mu \mathrm{m}$ (Sample A) and the fit of the Simmons model (the solid line). (c) The temperature dependence of resistance of Sample A junction. (d) The TMR dependence and magnetization curves of Sample A measured for the magnetizing field applied at the angle of $3^{\circ}$ with respect to the anisotropy axis.

dence of TMR. The current-voltage $(I-V)$ characteristics for the $\mathrm{NiFe} / \mathrm{Al}_{2} \mathrm{O}_{3} / \mathrm{Co}$ circular junction of $100 \mu \mathrm{m}$ in radius (referred to here as Sample A) with room-temperature resistance $R=7 \Omega$ are shown in Fig. 2(b). The experimental results (circles) are shown in Fig. 2(b) along with the best fit of the Simmons model ${ }^{13}$ (solid line) which gives the barrier thickness of $1.3 \mathrm{~nm}$ and the barrier height of $1.5 \mathrm{eV}$. The intrinsic junction resistance of Sample A slightly increased with the decrease of temperature, see Fig. 2(c), which suggests electron tunneling through the barrier as the operative transport process. The representative TMR dependence for Sample A measured at the field applied at an angle of $3^{\circ}$ with respect to the anisotropy axis is shown in Fig. 2(d). The maximal TMR decreased monotonically with the increase of the bias voltage and was one-half of its value at the bias of $0.3 \mathrm{~V}$, which was broadly consistent with other published data. ${ }^{1}$ The coupling fields and demagnetization fields extracted from simulated magnetization dynamics were less than 0.2 Oe for a typical sample. ${ }^{12}$

The layer of magnetic oxide was preformed on the surface of the bottom electrode (NiFe) before the layer of $\mathrm{Al}$ was deposited for the formation of $\mathrm{Al}_{2} \mathrm{O}_{3}$. ${ }^{12}$ This was achieved by the oxidation of the bottom magnetic electrode in 0.2 mbar of $\mathrm{O}_{2}$ inside the vacuum chamber for 4-6 h. The presence of this magnetic oxide layer was crucial to the effect described below and the junctions without such a layer did not show the effect.

In the key experiments of this study, the TMR of saturated tunnel junctions was measured in a rotating magnetic field of constant large amplitude $\left(H_{\text {ext }}=10^{3} \mathrm{Oe}\right)$ produced by a pair of rotating magnets. As the switching field is very small by comparison with the field $H_{\text {ext }}$ applied in these experiments, magnetizations of both electrodes follow the direction of the magnetic field. We estimate that the misalignment angle $\theta_{M}$ between magnetizations of the two electrodes (which appears to be due to the difference in the layer in-plane anisotropies) was less than $\theta_{M} \leqslant 10^{-2}$ rad for any orientation of the external field. Therefore, in the conventional magnetic tunnel junction one does not expect to obAIP license or copyright; see http://apl.aip.org/apl/copyright.jsp 


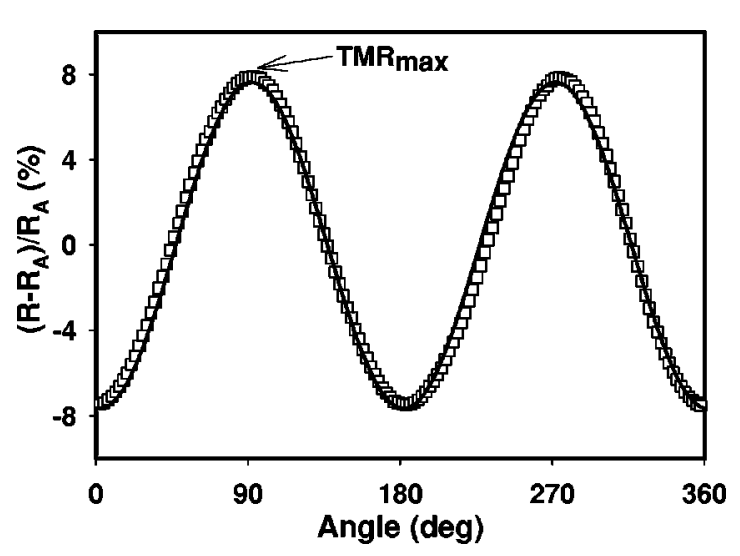

FIG. 3. TMR as a function of the angle between the external field $H_{\text {ext }}\left(10^{3} \mathrm{Oe}\right)$ and the axis of induced layer anisotropy for Sample A.

serve any TMR, as relative orientations of magnetizations of both electrodes are not significantly altered by the rotating field. The "conventional" angular change of $\mathrm{TMR}_{\mathrm{ang}}=[R(0)$ $\left.-R\left(\theta_{M}\right)\right] /\langle R\rangle$ caused by the misalignment of layer magnetizations can be roughly evaluated as ${ }^{6,14,15}$

$\mathrm{TMR}_{\text {ang }} \approx\left[G(0)-G\left(\theta_{M}\right)\right] /\langle G\rangle \approx P_{1} P_{2} \cdot \theta_{M}^{2} / 2$, see also Eq. (3) for $k_{\text {eff }}=0$. Hence, the ratio of angular TMR ang of the saturated tunnel junction to $\mathrm{TMR}_{\max } \approx 2 P_{1} P_{2}$ produced by the switching of the soft magnetic layer should be about $\mathrm{TMR}_{\text {ang }} / \mathrm{TMR}_{\max } \approx \theta_{M}^{2} / 4 \approx 10^{-3} \%$.

Surprisingly, this is not the case experimentally. Figure 3 shows the change in the resistance of Sample A as a function of the direction of the applied field $H_{\text {ext }}$. The change is normalized by the average sample resistance and the angle of the external field is taken with respect to the direction of the induced anisotropy. The observed change in the tunnel resistance of the saturated junction for Sample A, which we term here collinear TMR, is substantial $(16 \%)$. This variation of the resistance of the saturated junction is four orders of magnitude greater than the value expected by the conventional angular TMR dependence and of the same order as TMR due to the switching of magnetization of the soft magnetic layer $\mathrm{TMR}_{\max }=15 \%$.

The described collinear TMR has been observed for all studied junctions, irrespective of their geometrical form (circle, square, rhomb) and size, provided that the magnetic electrode was subjected to oxidation as described above. This rules out the shape anisotropy as the source of the phenomenon. Directions of the magnetic field in which the minimum resistance was observed $\left(0^{\circ}\right.$ and $180^{\circ}$ of Fig. 2 for Sample A) coincide with the directions of the in-plane magnetic anisotropy induced during junction fabrication. The collinear TMR is described well by the $\cos ^{2}$ function shown as the solid line in Fig. 3. Remarkably, the collinear TMR has a four-fold symmetry, i.e., the resistance is not affected by rotating the magnetization of the electrodes by $\pi$, and again this was the general feature of the phenomenon for the junctions of any shape.

Our results fall well in line with the qualitative model developed in this letter. We suggest that oxidation of the magnetic electrode results in the formation of thin magnetic oxide layer, which acts as the magnetic tunnel barrier. The collinear magnetoresistance appears when the (collinear) layer magnetizations are rotated by the external magnetic field with respect to the magnetization vector (or the antiferromagnetic vector in the case of the antiferromagnetic barrier) of the tunnel barrier (pinned by the anisotropy of magnetic dielectric). The angular dependency of the collinear TMR is consistent with the magnetic oxide layer being antiferromagnetic. Indeed, in the case of the antiferromagnetic tunnel barrier the rotation of quantization axis of the tunneling electrons by $\pi$ should be invariant with respect to the antiferromagnetic direction of the tunnel barrier and, the angle between the quantization axis corresponding to the maximum and minimum should be $\pi / 2$ (contrary to $\pi$ of the conventional junctions with the nonmagnetic barrier). This is similar to the angular dependence of tunneling between a ferromagnetic and an antiferromagnetic electrode. ${ }^{7}$ At $\phi$ $=1.6 \mathrm{eV}$ and $d=1.3 \mathrm{~nm}$, the theory requires an additional exchange energy of $J=0.02 \mathrm{eV}$ in order to explain the measured collinear TMR. We expect that the magnetic oxide formed in this experiment is most likely to be antiferromagnetic $\alpha-\mathrm{Fe}_{2} \mathrm{O}_{3}$, which is known to form predominantly at the interface of the oxidized permalloy (NiFe) films. ${ }^{16}$

It should be pointed that similarly, one expects to observe TMR in junctions with just one magnetic electrode and a magnetic dielectric layer. Another possible variation of this approach is to employ a junction with an antiferromagnetic electrode and ferromagnetic tunnel barrier.

The authors wish to thank Andre Geim for useful discussions, and Nick Fry and Phil Brown for technical assistance. The support of the EPSRC Grant No. GR/M23977/01 and Science Foundation Ireland SFI (Contract No. 00/PI.1/Co42) is acknowledged.

${ }^{1}$ J. S. Moodera, L. R. Kinder, T. M. Wong, and R. Meservey, Phys. Rev. Lett. 74, 3273 (1995).

${ }^{2}$ S. Gider, B. U. Runge, A. C. Marley, and S. S. P. Parkin, Science 281, 797 (1998).

${ }^{3}$ J. M. de Teresa, A. Barthélémy, A. Fert, J. P. Contour, F. Montaigne, and

P. Seneor, Science 286, 507 (1999).

${ }^{4}$ S. Yuasa, T. Nagahama, and Y. Suzuki, Science 297, 234 (2002).

${ }^{5}$ H. Yang and A. R. Smith, Phys. Rev. Lett. 89226101 (2002).

${ }^{6}$ J. Slonczewski, Phys. Rev. B 39, 6995 (1989).

${ }^{7}$ A. A. Minakov and I. V. Shvets, Surf. Sci. 236, L377 (1990).

${ }^{8}$ N. Berdunov, S. Murphy, G. Mariotto, and I. V. Shvets, Phys. Rev. Lett. 93, 057201 (2004).

${ }^{9}$ G. Mariotto, S. Murphy, and I. V. Shvets, Phys. Rev. B 66, 245426 (2002).

${ }^{10}$ X. Hao, J. S. Moodera, and R. Meservey, Phys. Rev. B 42, 8235 (1990).

${ }^{11}$ P. LeClair, J. K. Ha, H. J. M. Swagten, J. T. Hohlhepp, C. H. van deVin, and W. J. M. de Jonge, Appl. Phys. Lett. 80, 625 (2002).

${ }^{12}$ A. N. Grigorenko and D. J. Mapps, J. Phys. D 36, 791 (2003).

${ }^{13}$ J. C. Simmons, J. Appl. Phys. 34, 2581 (1963).

${ }^{14}$ R. Landauer, IBM J. Res. Dev. 1, 223 (1957); M. Büttiker, Phys. Rev. Lett. 57, 1761 (1986).

${ }^{15}$ H. Jaffres, D. Lacour, F. N. Van Dau, J. Briatico, F. Petroff, and A. Vaurès, Phys. Rev. B 64, 064427 (2001).

${ }^{16}$ C. H. Bajorek, M.-A. Nicolet, and C. H. Wilts, Appl. Phys. Lett. 19, 82 (1971). 